\title{
Las herramientas 2.0 en la escritura académica: buscadores y diccionarios
}

\author{
Tools 2.0 in Academic Writing: Search Engines and Dictionaries
}

\section{As ferramentas 2.0 na escritura acadêmica: buscadores e dicionários}

\author{
Montserrat Casanovas Catalá ${ }^{1}$
}

\section{Resumen}

En este artículo se exponen algunos de los resultados de un estudio sobre el uso de las tecnologías de la información y la comunicación (TIC) en la escritura académica que está llevando a cabo el Grupo de Investigación en Mediación Lingüística (GııL) de la Universidad de Lleida. Dicho estudio pretende mostrar cómo se integran herramientas como los diccionarios en línea o los buscadores en el proceso de elaboración de textos académicos por parte de estudiantes universitarios.

Palabras clave: Escritura académica, textos académicos, TIc, herramientas web 2.0.

\section{Abstract}

This article presents some of the results of a study based on the use of Information and Communication Technologies (ICT) in academic writing, which is being conducted by the Research Group in Linguistic Mediation of the University of Lleida. This study analyzes the role that ICT plays in the process of writing academic texts and aims to show how specific tools such as online dictionaries or search engines are integrated in the immersion process of academic texts for the university student demography.

\section{Keywords: Academic writing, academic texts, ICTs, web 2.0 tools.}

\section{Resumo}

Neste artigo se expõem alguns dos resultados de um estudo sobre o uso das tecnologias da informação e comunicação (TIC), na escritura acadêmica que está levando a cabo o "grupo de Investigación en Mediación Lingüística" (GIML) da "Universidad de Lleida". 0 estudo procura mostrar como se integram ferramentas como os dicionários online, ou os buscadores no processo de elaboração de textos acadêmicos por parte de estudantes universitários.

Palavras-chave: Escritura acadêmica, textos acadêmicos, TIc, ferramentas web 2.0.

Artículo recibido el 10 de marzo de 2015 y aprobado el 26 de junio de 2015

1 Universidad de Lleida, Lleida, España. Correo electrónico: m.casanovas@didesp.udl.cat 


\section{Introducción}

Desde hace unos años, Internet se ha convertido en un elemento clave en muchos aspectos de nuestra vida, personal y académica. Las herramientas web 2.0 han supuesto, como se ha señalado (Leu, Kinzer, Coiro y Cammack, 2004; Knobel y Lankshear, 2014), el paso de un contexto comunicativo plano y unimodal (en papel, con un receptor y un emisor y un mensaje en un único soporte) a un entorno multimodal, en el que la multialfabetización resulta clave para poder comunicarse de manera eficaz (Nuzzaci, 2013). Ello implica que escribir, leer y aprender en Internet comporta un nuevo modo de explorar la realidad y construir el conocimiento (Coiro, 2003), que requiere de competencias que van más allá de la concepción tradicional de la literacidad (Ballano y Muñoz, 2010; Cassany, 2011; Coiro, 2003; Leu, Kinzer, Coiro y Cammack, 2004; Wilber, 2009).

La Red es un contenedor en constante transformación, en el que convergen múltiples elementos. Es, como comentan Area y Pessoa (2012, p. 14), "simultáneamente, una biblioteca universal, un mercado global, un gigantesco puzzle de piezas informativas conectadas hipertextualmente, una plaza pública de encuentro y comunicación de personas que forman comunidades sociales, es un territorio donde prima la comunicación multimedia y audiovisual, así como la diversidad de entornos virtuales interactivos". En este marasmo de opciones, se han de mover los actuales estudiantes universitarios para poder elaborar, con éxito, sus textos académicos y desarrollar satisfactoriamente todas las fases de elaboración textual, desde la búsqueda y selección de la información hasta la corrección del texto final.

Esta contribución pretende abordar una cuestión relacionada, precisamente, con la inclusión de las herramientas web 2.0 en el contexto académico: el uso de las Tic en el proceso de elaboración de textos académicos por parte de estudiantes universitarios ${ }^{2}$ $y$, en concreto, el empleo que dichos estudiantes

2 Este estudio se está llevando a cabo desde el Grupo de Investigación en Mediación Lingüística (GIML) de la Universidad de Lleida. Para más información sobre el GIML puede consultarse su página web (http://www.giml.udl.cat) y su Twitter (@GIMLUdL). hacen de los buscadores y de las herramientas de revisión y corrección textual.

\section{Metodología}

\section{Objetivos}

Como decíamos, nuestro estudio se centra en el uso de las TIC en el contexto universitario. Desde hace unos años, la relación entre las TiC y los estudiantes universitarios se ha explorado desde el examen de las tecnologías que usan los estudiantes en su vida diaria, con especial atención a las redes sociales y la presencia (o más bien la ausencia) de estas en sus actividades académicas y/o en los planes de estudios (Gutiérrez, Palacios y Torrego, 2010; Tapia, Gómez, Herranz de la Casa y Matellanes, 2010; Espuny, González, y Gisbert, 2011; Monge y Olabarri, 2011). $\mathrm{El}$ análisis que presentamos viene a complementar esta perspectiva, puesto que busca analizar cómo se integran las tecnologías web 2.0 en el proceso de redacción de textos académicos, respondiendo a varias preguntas específicas, como qué instrumentos se emplean y por qué y cómo se utilizan. Esta investigación parte de los fundamentos teóricos de los nuevos estudios de literacidad ${ }^{3}$ (NEL), ámbito que propone un concepto de literacidad centrado en su componente social y alejado de la visión psicológica de la escritura, iniciada a principios de los años setenta del siglo pasado (Emig, 1971, 1983).

El estudio combina el análisis de datos cuantitativos, recogidos mediante un cuestionario, $\mathrm{y}$ cualitativos, documentados a través de grupos de discusión, en fase de diseño. En este artículo vamos a ocuparnos de la exploración de algunos de los primeros, examinados desde el punto de vista de la estadística descriptiva. En concreto, analizaremos los datos obtenidos sobre el perfil tecnológico de los entrevistados, por un lado, y sobre las herramientas

3 Del inglés New Literacy Studies. La rápida introducción de las tecnologías digitales ha conllevado un rosario de denominaciones propuestas para la literacidad digital desde el ámbito anglosajón, como ICT literacy, information technology literacy, media literacy, net literacy, online literacy o multimedia literacy. Puede encontrarse una revisión de esta terminología en Oliver y Tomei (2000) y Markauskaite (2006). 
digitales empleadas en la búsqueda de información y para la revisión de los textos, por otro.

\section{Técnica de recogida de datos: el cuestionario}

Como comentábamos más arriba, para la recogida de datos cuantitativos se diseñó un cuestionario, denominado NLits. Está estructurado en dos partes, además de un apartado introductorio en el que se expone su propósito y procedencia:

a. perfil del encuestado, en la que se incluyen 4 preguntas sobre el perfil social del entrevistado y 4 sobre su perfil tecnológico y

b. el cuestionario propiamente dicho, que consta de 25 ítems.
En él se abordan cuestiones relacionadas con las diversas fases asociadas al proceso de elaboración de textos:

1. búsqueda de información,

2. selección de esa información,

3. planificación,

4. ejecución y

5. corrección y revisión textual.

Las preguntas relativas a cada una de estas fases pretenden arrojar luz sobre las herramientas - digitales o analógicas - a las que recurren los estudiantes, cómo las usan y por qué las han elegido. Véase un ejemplo de pregunta en la imagen siguiente:

\section{1. ¿Qué haces habitualmente cuando escribes un trabajo en castellano y tienes una duda léxica, de expresión o de sintaxis?}

a) Utilizo un diccionario en papel.

b) Utilizo un diccionario en línea.

c) Escribo la palabra o la expresión directamente en el buscador (Google u otros).

d) Utilizo las herramientas de revisión del procesador de texto.

Imagen 1. Ejemplo de pregunta en el cuestionario NLits

El cuestionario fue validado por diversos expertos procedentes, básicamente, de dos campos: la didáctica de la lengua y la tecnología.

\section{Muestra}

después de la elaboración del cuestionario, se hacía necesario su pilotaje, con el fin de testar su funcionamiento y observar qué tendencias se documentaban para diseñar y perfilar otros instrumentos que nos permitieran analizar el objeto de estudio.

Para dicho pilotaje, recogido en Campos (2013), se seleccionó una muestra constituida por 41 estudiantes del Grado en Educación Primaria de la Universidad de Murcia, que voluntariamente se ofrecieron a participar ${ }^{4}$. Los datos se recogieron en el mes de mayo de 2013.

El perfil social de la muestra es el esperable de acuerdo con la población en estudio, ya que la mayoría de los informantes son jóvenes, con una media de edad de 21 años, y predominan las mujeres (68,3\%), como es habitual en las titulaciones de Magisterio. En cuanto a los estudios realizados, el $47,5 \%$ ha accedido a la formación superior después de cursar bachillerato, el 27,5\% proviene de otros estudios universitarios y el $25 \%$ restante, de ciclos formativos de grado superior, es decir, de formación profesional. El castellano es la lengua materna de la mayoría de ellos $(97,5 \%)$. Socialmente, corresponden,

4 Los estudiantes eran de primer curso. Al ser un estudio piloto, el objetivo no era obtener una muestra representativa y los encuestados suponen un $54,6 \%$ del total del grupo (75 estudiantes). 
pues, al estándar esperable en la tipología de estudiantes que se analiza.

\section{Resultados}

\section{Perfil tecnológico}

El perfil tecnológico de los encuestados se ha definido a partir de la información recogida en las cuatro preguntas situadas en la sección primera del cuestionario, como decíamos. De esos cuatro ítems, que pretenden documentar la competencia digital general de los encuestados, dos buscan hallar la frecuencia con la que los informantes se relacionan con la tecnología en su vida personal y académica (empleo y frecuencia de uso de medios digitales), mientras que los dos restantes les preguntan sobre sus hábitos - digitales o analógicos- en el aula (toma de apuntes con o sin ordenador y gestión de las aplicaciones del ordenador). Creemos que esta información es básica para, posteriormente, poder correlacionar los perfiles digitales generales obtenidos con el comportamiento específico en el ámbito académico.

Si analizamos los datos recogidos en cuanto a las herramientas tecnológicas que dicen usar los encuestados no sorprende encontrar la aplicación de comunicación instantánea WhatsApp ${ }^{5}$ en primer lugar, empleada, como puede observarse en el gráfico 1, por un $90 \%$ de los informantes, si sumamos aquellos que dicen utilizarla siempre $(87,5 \%)$ y los que lo hacen frecuentemente $(2,5 \%)$. Las redes sociales se sitúan en segundo lugar y son usadas también por el $90 \%$ de los entrevistados, aunque con una frecuencia algo menor pues son menos los estudiantes que dicen utilizarlas siempre $(57,5 \%)$. El resto de las herramientas se sitúan muy lejos de esos porcentajes. Así, por ejemplo, dicen emplear, frecuentemente o siempre, los blogs un 23,5\% de los entrevistados; los lectores de feeds RSS, un 12,8\% de ellos y Skype solamente el $15 \%$.

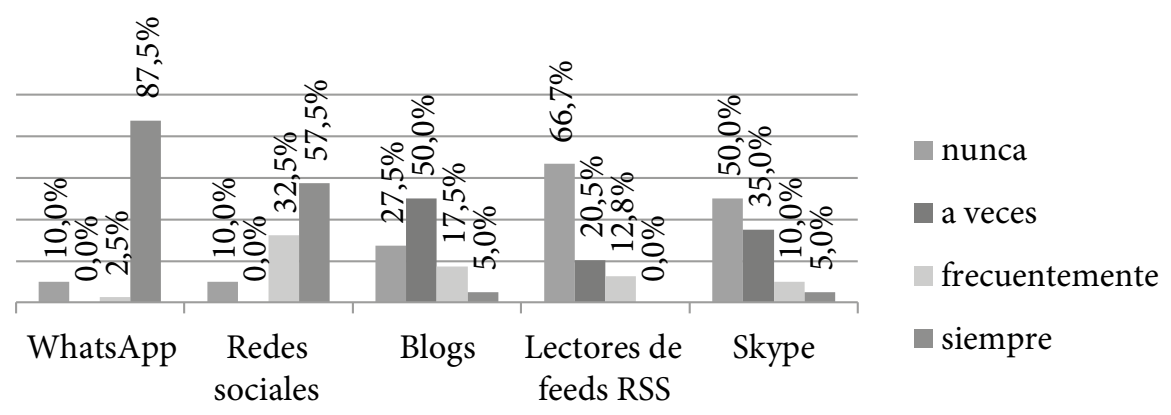

Gráfico 1. Frecuencia de uso de herramientas digitales

Las herramientas 2.0 están, pues, presentes en la vida de los informantes, aunque, por su tipología

5 Según la Fundación Telefónica (2014), esta es la aplicación de mensajería instantánea más empleada en España, con un 98,5\% de usuarios de ese tipo de mensajería que lo emplean. El estudio también señala que en el $96 \%$ de las conversaciones se emplea, de un modo u otro, mensajería, indicio de cómo se transforman los hábitos conversacionales con la introducción de las Tic. Una de las cuestiones, desde nuestro punto de vista más relevantes, es el papel que van a tener las tecnologías móviles (dispositivos y apps) en el aprendizaje de lenguas, cuya introducción en las aulas ya empieza a ser visible de la mano del Mobile-Assisted Language Learning (MALL). Véase Burston (2013) para una revisión sobre el maLl.
(WhatsApp y redes sociales) podemos presumir que se circunscriben más a su ámbito personal o vernáculo ${ }^{6}$. Ello corroboraría los resultados hallados en otros estudios, según los cuales la tecnología desempeña en la vida personal un papel mucho más relevante que en su esfera académica (Ballano y Muñoz, 2010; Cassany, 2011; Ng, 2012).

6 Empleamos el adjetivo "vernáculo" en el sentido de Cassany (2011, p. 92), quien lo define como aquellas prácticas que incluyen "tareas letradas que ocurren en el ámbito privado y ocioso de la familia y los amigos, que hacemos por iniciativa propia, cuando y como nos da la gana y sin seguir norma o directriz alguna". 
También parecen refrendar tal afirmación los resultados hallados por lo que se refiere a la segunda pregunta del cuestionario relativa a los hábitos de empleo de los medios tecnológicos en el aula. A pesar del extendido uso social de la tecnología, solamente el 5,1\% de los encuestados, como puede observarse en el gráfico 2, toma los apuntes con ordenador o con otros dispositivos digitales. La mayoría de ellos, un $69,2 \%$, son, en este sentido, "analógicos", mientras que un $25,6 \%$ indica emplear ambas posibilidades indistintamente.
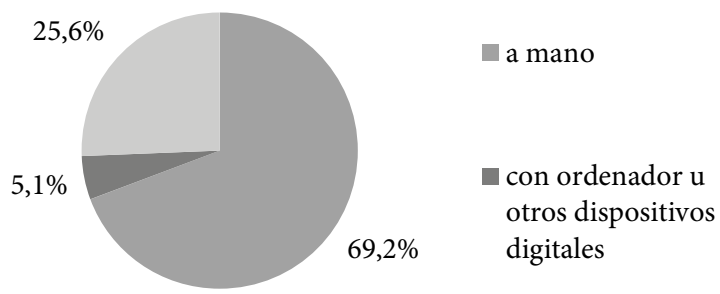
otros dispositivos digitales

Gráfico 2. Procedimientos de toma de apuntes
Se detecta, pues, que, a pesar de las ventajas que podría tener la toma de apuntes digital (rapidez, posibilidad de manipular el texto, etc.), se sigue prefiriendo el papel. En cuanto a los encuestados que afirman que emplean medios digitales o analógicos indistintamente, este es uno de los comportamientos que interesa examinar con más profundidad en futuros análisis, de cariz más cualitativo, en el sentido de detectar en qué consiste esa alternancia entre lo digital y lo analógico, por lo que a la toma de datos en el aula se refiere.

Igualmente, se ha preguntado por la gestión de los dispositivos electrónicos cuando se usan. Los resultados, como puede verse el gráfico 3, parecen indicar que no son muchos los estudiantes que complementan su toma de apuntes electrónica con otras herramientas, como podría ser el campus virtual de la universidad.

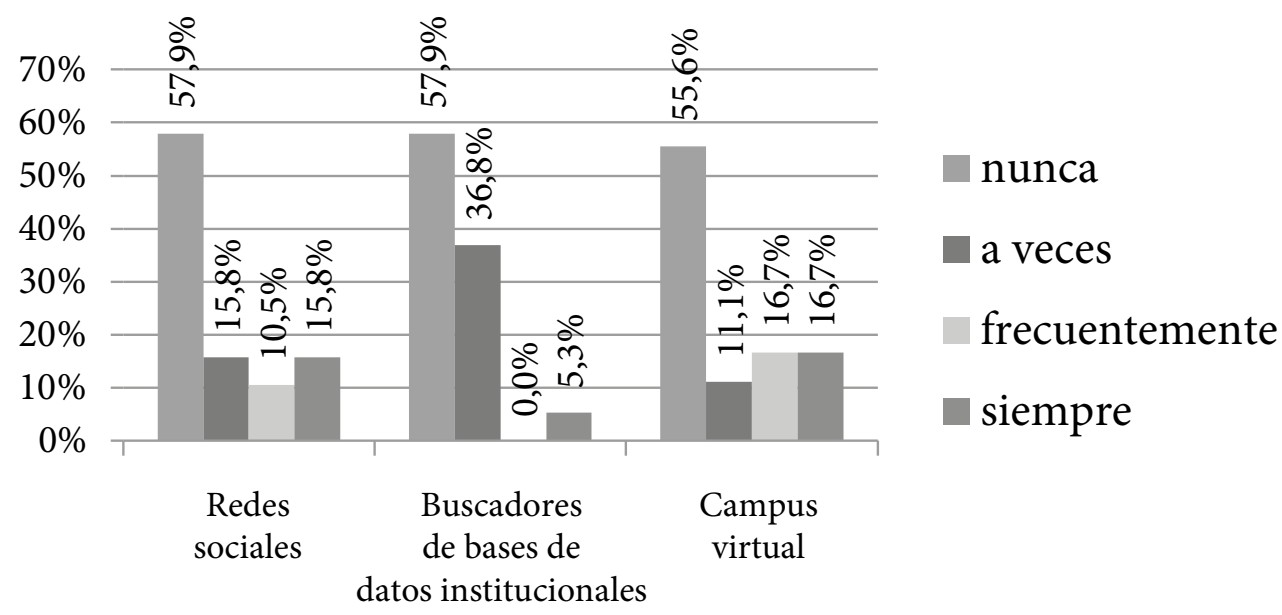

Gráfico 3. Uso de herramientas digitales mientras toman apuntes con dispositivos digitales

El análisis cualitativo también podrá ofrecer más datos respecto a este comportamiento, con el afán de documentar esas capacidades de multitarea que algunos estudios han recogido ${ }^{7}$. Debemos tener en cuenta, además, que en este estudio se recogen las afirmaciones de los informantes sobre su comportamiento y no se documenta de manera directa dicho

7 Véase Dede (2005), por ejemplo. comportamiento, por lo que sospechamos que algunos hábitos, como, por ejemplo, el uso de las redes sociales mientras se está en el aula, no se reconocen porque los informantes se autocensuran.

Estos dos datos parecen describir, pues, un perfil de estudiante que sí ha integrado las TIC en su vida personal y se comunica mediante los diferentes mecanismos que tiene a su alcance (WhatsApp, redes sociales, etc.), pero que es más analógico de 
lo que podría pensarse, a priori, en el ámbito académico, en el que muestra unos hábitos (escritura manual, procedimientos lineales, etc.) no muy distintos de las generaciones anteriores.

Veamos a continuación qué correlato tienen estos datos con la composición de textos.

\section{Uso de las tic en la composición de textos}

La segunda cuestión que nos ocupa es el uso de las TIC en la composición de textos académicos. En el estudio se examinan qué herramientas se emplean, cómo se escogen y usan y cuáles son los criterios de su elección en las distintas fases de la elaboración del texto, es decir, en la búsqueda y selección de información, en la planificación del trabajo y en su ejecución, así como en su revisión. En este artículo vamos a ocuparnos, como se ha comentado, de los mecanismos y herramientas empleados en la gestión de la información, por una parte, y aquellos usados en la revisión textual, por otra.

\section{Gestión de la información}

En primer lugar, para comprender cómo se enfrentan los estudiantes a la gestión de los textos, nos interesa hallar datos sobre cómo obtienen la información necesaria para elaborarlos. La importancia de esta fase ha sido puesta en relevancia por numerosos autores, quienes han señalado las dificultades con las que se encuentran los estudiantes universitarios a la hora de seleccionar las fuentes y de procesar la información obtenida. Dichas dificultades se deben al paso de un modelo de confección textual en la Educación Secundaria en el que básicamente solo se les ofrece una fuente de información a estudios universitarios que les exigen barajar fuentes múltiples y en diversos formatos (Castelló, 2009).

Por lo que se refiere al tipo de fuentes consideradas, se documentan tres tipos de comportamientos, como puede observarse en el gráfico 4. Primero, se detectan los estudiantes que acuden únicamente a fuentes digitales, grupo que representa el 48,8\% de los entrevistados. El segundo segmento lo conforman aquellos que dicen utilizar medios tanto digitales como impresos, que suponen el 46,3\%, mientras que en mucho menor porcentaje, $4,9 \%$, se encuentran quienes exclusivamente recurren a materiales impresos.

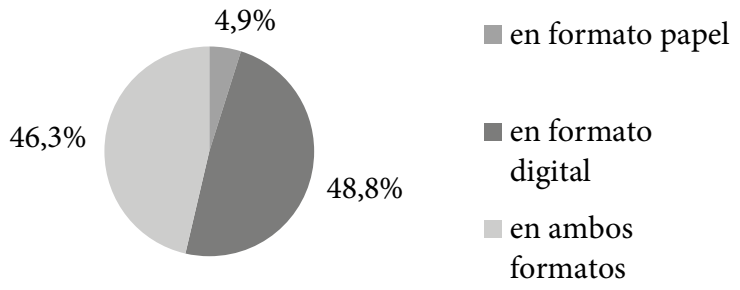

Gráfico 4. Tipos de fuentes bibliográficas consultadas

Esta presencia de lo digital nos lleva a deducir que los estudiantes ya no consideran como primer origen de información para la elaboración de trabajos académicos la biblioteca de la universidad o del municipio, sino que ven Internet como el repositorio de datos por excelencia y acuden a ella, ya sea como fuente única o como complemento a la información en papel.

$\mathrm{Al}$ ahondar en los comportamientos de los estudiantes en el caso del uso de Internet como herramienta de búsqueda de información, hallamos un dato que consideramos muy significativo. Como se observa en el gráfico 5, la mayoría de los encuestados (61\%) dice emplear siempre buscadores genéricos. Si a ello se suma la cifra de los que dicen hacerlo frecuentemente, que es el 31,7\%, llegamos hasta el $92,7 \%$ de la población encuestada que muestra ese hábito de búsqueda. El uso regular de buscadores especializados según la temática del estudio que tenga que realizarse o de bases de datos científicas queda relegado al $31,7 \%$ y al $12,5 \%$, respectivamente, si incluimos los estudiantes que dicen usarlos siempre y frecuentemente. Un $60 \%$ de los estudiantes dice que nunca emplea bases de datos científicas. 


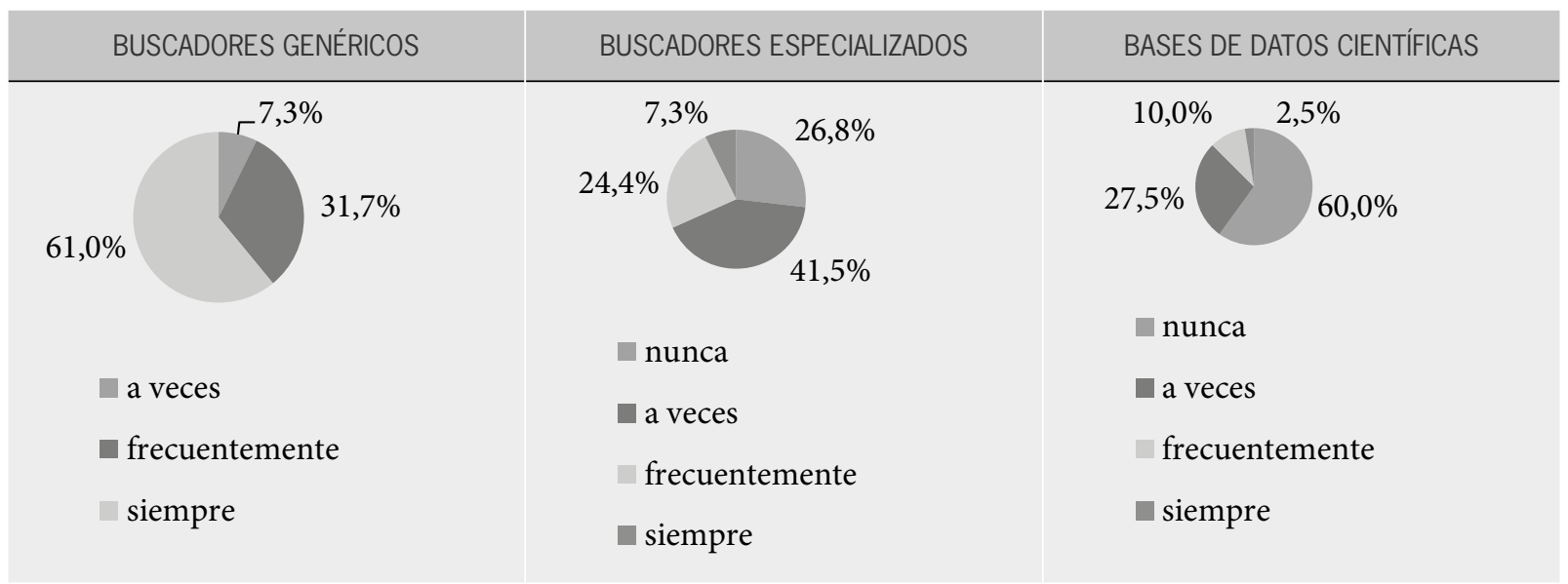

Gráfico 5. Frecuencia de uso de distintos tipos de buscadores

Parece, pues, como otros autores han indicado (Monereo y Fuentes, 2005), que el estudiante recurre al primer sistema de búsqueda que se tiene a mano, que es el que emplearía en su vida cotidiana para solucionar cuestiones como hallar el número de teléfono de una tienda de móviles, por ejemplo. Es decir, no se plantea una selección del tipo de fuente de acuerdo con el objetivo académico que se le propone ni se considera que es necesario acudir a lugares especializados para optimizar cuantitativa y cualitativamente la búsqueda. Los estudiantes simplemente trasvasan aquellas competencias de búsqueda que les son útiles en la vida diaria a su quehacer académico. Resulta interesante plantearse por qué no se han desarrollado estrategias propias en este caso. Desde nuestro punto de vista, la falta de formación específica es clave. Aunque no disponemos de datos sobre la formación que han recibido los universitarios respecto a la gestión de información en Internet en España, lugar de toma de los datos, una cifra que puede ser indicativa, salvando las distancias, es la que ofrecen Tolar y Chadwick (2000), en un estudio sobre los criterios que emplean los estudiantes universitarios estadounidenses al evaluar las fuentes de información de Internet y de las bibliotecas. Los autores citados documentan que, mientras que un $60 \%$ de los estudiantes ha recibido formación en el uso de los recursos de las bibliotecas, solamente un $30 \%$ lo ha hecho respecto al uso de Internet como fuente de información académica.
Ello va en la línea de los datos recogidos por Iglesias y Molina (2013) en España, quienes detectaron que los estudiantes sabían dónde debían buscar la información y cómo incorporarla a sus trabajos académicos, pero "carecían de habilidades para enfrentarse de forma eficaz a la propia búsqueda de información $y$, sobre todo, para registrarla e incorporarla de forma ética en dichos trabajos" (Iglesias y Molina 2013, p. 53) ${ }^{8}$.

Esa falta de competencia en gestión de la información se ve reflejada en las producciones elaboradas por los alumnos, como recogen algunos estudios. Así, por ejemplo, Ballano y Muñoz (2010, p. 5) ${ }^{9}$, documentan el ejemplo de un estudiante que en la bibliografía había incluido una página web sobre una noticia acerca de una exposición de pintura en Argentina. El estudiante, que debía realizar un estudio sobre poesía social, había llegado a ese recurso porque el título de la noticia era "Sensibilidad social: intimismo y poesía”. Las palabras clave de búsqueda que había empleado ("poesía" y "social"), en un buscador genérico, le habían llevado, pues, a esa página y la labor de discriminación había sido nula.

8 Las autoras recogieron los datos a partir del Cuestionario sobre gestión de la información (GESTINF), aplicado a 824 estudiantes de primer y tercer curso de Magisterio de la Universidad de Oviedo en España en los cursos 2008/2009 y 2009/2010.

9 En esta investigación se analiza un corpus de 48 trabajos realizados durante los cursos 2006-07 y 2007-08 por universitarios de primer año de Filología de la Universidad de Deusto (España). 
Comportamientos como los señalados pueden ser indicativos, como se ha comentado, de la falta de competencia digital específica (Ng, 2012; Togia y Tsigilis, 2010; Khan, Bhatti y Khan, 2011) que poseen los estudiantes y de la brecha que existe entre las competencias digitales sociales y las académicas (Moje, 2000; Cassany, 2011; Ware y Warschauer, 2005).

\section{Revisión textual}

Por lo que se refiere a los procedimientos y herramientas de revisión textual, nos interesa, en primer lugar, conocer qué tipo de instrumentos dicen emplear los encuestados a la hora de solventar las dudas lingüísticas que se plantean en el proceso de elaboración de un texto. Hemos preguntado, en el cuestionario, sobre su proceder en dos casos: cuando se escribe en español, que, recordemos, es la lengua materna de la mayoría de los entrevistados; y cuando se hace en una lengua extranjera, el inglés en este caso.

Veamos qué sucede en cada contexto:

Se han documentado, como se recoge en el gráfico 6 , tres procedimientos comunes a la escritura en lengua propia y en lengua extranjera: acudir a un diccionario en papel, consultar un diccionario en línea y emplear un buscador. Se detecta una cuarta posibilidad, recurrir a las herramientas del editor de texto, que dicen los encuestados emplear solamente en el caso de escribir en el idioma materno. Veamos qué sucede en cada caso.

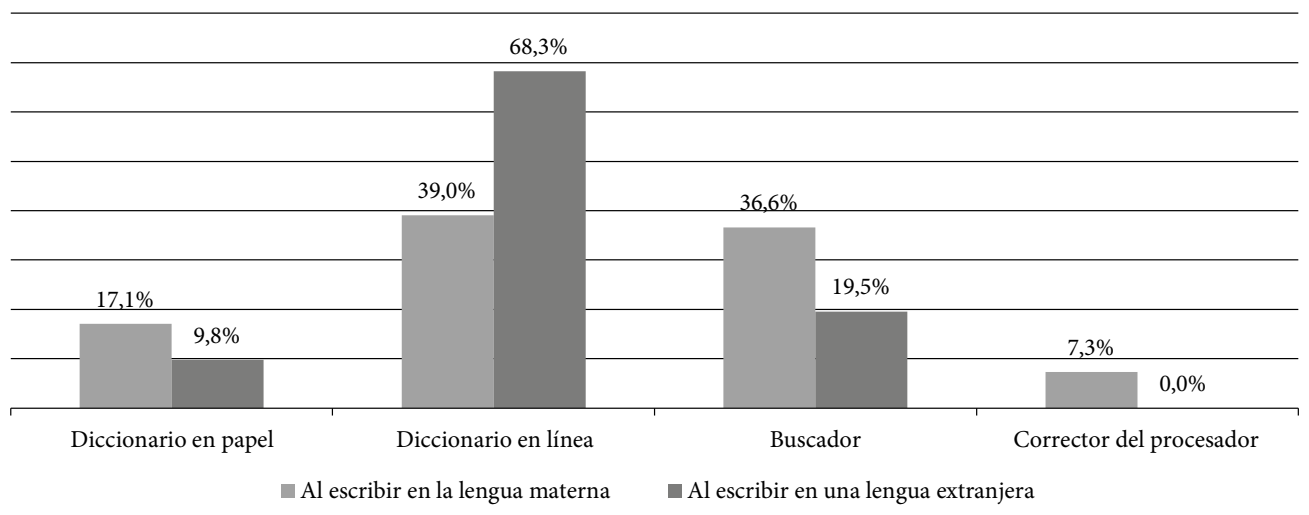

Gráfico 6. Herramientas usadas para la consulta de dudas lingüísticas

En cuanto a la escritura en español, si analizamos de menor a mayor frecuencia los procedimientos detectados, observamos que los informantes acuden en menor medida $(7,3 \%)$ a las herramientas de que dispone el editor de textos empleado, mientras que el segundo lugar lo ocupan los diccionarios en papel $(17,1 \%)$. Los datos de uso de buscadores $(36,6 \%)$ y de diccionarios en línea (39\%) están muy próximos y su suma supone el 75,6\% de las respuestas. Se detecta, pues, que las herramientas web son las mayoritarias.

Por lo que se refiere al uso de buscadores, si tenemos en cuenta las cifras presentadas en el gráfico 5, que indican que el 92,7\% de los informantes usan siempre o frecuentemente buscadores genéricos, podemos formular la hipótesis de que, en el caso de pretender resolver dudas lingüísticas, el usuario acudirá a ese tipo de buscadores y no se planteará el uso de plataformas especializadas para la búsqueda de información gramatical, por ejemplo. Es decir, se empleará el mismo procedimiento para saber qué significa una palabra o qué régimen tiene un verbo que para obtener información necesaria en la vida cotidiana.

Ello sería, de nuevo, indicativo de esa falta de competencia digital específica que comentábamos más arriba y de la brecha entre las competencias digitales sociales y académicas que poseen los estudiantes universitarios. 
En el caso de escritura en una lengua extranjera, resulta interesante destacar que los porcentajes son distintos por lo que se refiere a los procedimientos, podríamos decir, no lexicográficos. Así, no se considera la opción de resolver las dudas a partir de las herramientas del procesador (frente al 7,3\% que sí lo hace, como decíamos, cuando se escribe en la lengua materna) y acude a un buscador únicamente un 19,5\% de los encuestados, un 17,1\% menos que en el caso de la lengua materna. Aunque esta es una cuestión que necesita de un tratamiento más profundo y es uno de los elementos que va a abordarse en los grupos de discusión, quizá sea la variable dominio de la lengua la responsable de esa variación y tal vez cuanto menor sea el dominio de un idioma, más se acuda a las obras, en papel o en línea, que ofrecen a priori una mayor fiabilidad en sus respuestas y en las que la información lingüística que se incluye es más completa (derivación verbal, pronunciación, etc.). Ello viene corroborado por el porcentaje de encuestados que dicen emplear el diccionario que, en el caso de una lengua extranjera es del $78,1 \%$, frente al $56,1 \%$ de los informantes que lo usaban en la escritura en primera lengua, como se ha comentado.

En cuanto a la distribución entre obra en papel o en línea, son mayoría los estudiantes que emplean diccionarios electrónicos, la herramienta más utilizada tanto en el caso de redacción en lengua materna como extranjera, aunque los porcentajes de uso son distintos: el 17,1\% de los informantes dice acudir a un diccionario en papel cuando redacta en su lengua materna, frente al $39 \%$ que recurre a un diccionario en línea; mientras que en el caso de escritura en una lengua extranjera, el 9,8\% acude al papel, frente al $68,3 \%$ que consulta una obra en línea.

Creemos que la preferencia por la versión electrónica en el caso de una lengua extranjera iría en consonancia con nuestra hipótesis de la influencia del grado de domino de la lengua, en el sentido de que los diccionarios electrónicos ${ }^{10}$ contienen, amén

10 Aunque no podemos dedicarnos a ello en esta contribución, una cuestión muy relevante en este caso es el análisis de la calidad de los diccionarios, compendios lexicográficos y demás herramientas similares que aloja Internet, dado que la tipología de estas herra- de la información lexicográfica de los compendios analógicos, elementos multimediales, como por ejemplo la pronunciación de un término, y cooperativos, como los foros de discusión, ${ }^{11}$ a los que el usuario puede acudir para resolver dudas concretas.

\section{Conclusiones}

este estudio exploratorio, aunque por la cantidad de la muestra recogida sus resultados no puedan extrapolarse a toda la población en estudio, sí ofrece datos interesantes respecto a algunos de los comportamientos en relación al uso de las Tic de los estudiantes universitarios.

En primer lugar, por lo que se refiere al perfil tecnológico de los encuestados, se detecta un uso social de la tecnología muy extendido, sobre todo de las herramientas de mensajería instantánea y redes sociales. La integración de esa tecnología en la vida académica parece ser parcial, puesto que se emplean, en general, de manera limitada en la vida diaria académica. Ello iría en el sentido de otras investigaciones, como la de Gómez, Roses y Farias (2012), quienes han detectado que los estudiantes universitarios usan intensivamente las redes sociales en su vida diaria ("están prácticamente 'conectados' durante todo el día" (Gómez, Roses y Farias, 2012, p. 135), pero que el uso académico de esas redes es escaso.

En segundo lugar, en cuanto a la gestión de la información, se ha recogido información respecto a dos cuestiones esenciales: el soporte al que se acude para la búsqueda de información y la tipología de herramientas tecnológicas que se emplean para la búsqueda. En cuanto al primer elemento, se recogen tres tipos de comportamientos: 1) usuarios digitales, los que solamente acuden a fuentes de Internet, 2) usuarios analógicos, que emplean únicamente

mientas es muy variada y va desde las obras de muy contrastada solvencia, como los diccionarios de la RAE y similares, a compendios realizados sin ningún criterio lexicográfico. Varios autores se han dedicado en los últimos años a ello.

11 El uso de los foros es, desde nuestro punto de vista, un elemento clave a la hora de analizar la inclusión de la tecnología en los procesos de redacción. Este va a ser uno de los elementos que vamos a examinar con mayor atención en el estudio cualitativo. 
fuentes impresas y 3) usuarios mixtos, que acuden a instrumentos digitales e impresos. En el caso del tipo de instrumentos empleados, se documenta que los buscadores genéricos son los más utilizados a la hora de recoger información para elaborar un texto, seguidos por los buscadores especializados y, a mucha distancia, las bases de datos especializadas.

En tercer lugar, el análisis de los procedimientos de revisión textual que dicen llevar a cabo los estudiantes pone de relieve que el instrumento más empleado es el diccionario en línea, seguido del buscador. Se detecta una diferencia sustancial entre el comportamiento en el caso de escritura en lengua propia, en el que se incorpora el uso del corrector del procesador de textos, y en el de lengua extranjera, caso en el que se acude con más frecuencia a diccionarios.

Como planteábamos en otro lugar (Casanovas, 2014), ello nos lleva a postular la existencia de dos patrones ala hora de confeccionar un texto académico, por lo que a inclusión de las herramientas web 2.0 en la redacción de textos académicos se refiere. Por una parte, el estudiante de herramientas inmediatas, es decir, aquel que, frente a un texto, emplea las herramientas que ya conoce para otras labores que no son necesaria ni estrictamente académicas, como los buscadores genéricos y las fuentes de información no especializadas, de base principalmente digital, es un "redactor googleizado" (Casanovas, 2014), porque para él, el buscador es el santo grial que ha de ayudarle tanto en el proceso de búsqueda y selección de información como en la revisión textual. Por otro lado, el estudiante de herramientas integradas es consciente de que se enfrenta a una tarea académica y que ello requiere herramientas más sofisticadas y acordes con su complejidad, como diccionarios y recursos lingüísticos específicos, tanto digitales como analógicos. Por ello, acude a instrumentos que considera más útiles y/o fiables: bases de datos especializadas, diccionarios, foros, etc.

A partir de este estudio, se pone de relieve que es necesario recoger evidencias empíricas que permitan ahondar en los comportamientos actuales de los estudiantes, para conocer cómo integrar las habilidades y capacidades que estos pueden aportar, transferidas de su "vida vernácula digital", rica en herramientas y en medios. Datos como qué herramientas concretas se emplean en la elaboración textual y cuáles son los criterios que llevan a tal selección han de ayudar a diagnosticar de qué punto ha de partirse a este respecto.

Así pues, el análisis nos lleva a plantearnos más interrogantes sobre la integración de las herramientas web 2.0, relacionadas con cuestiones más instrumentales (¿cómo se seleccionan las herramientas para la corrección textual?, ¿qué papel desempeñan los foros en los procesos de corrección?) y otras más competenciales (¿cómo ayudar a superar la brecha entre las competencias vernáculas y académicas?). Estas y otras cuestiones han de llevarnos a ayudar al diagnóstico de en qué punto de "alfabetización digital" se hallan los estudiantes universitarios y cómo deberíamos afrontar su formación para realmente garantizar que las competencias tecnológicas se integren como tales en su formación universitaria.

\section{Referencias}

area, M. y Pessoa, T. (2012). De lo sólido a lo líquido: las nuevas alfabetizaciones ante los cambios culturales de la Web 2.0. Comunicar, 38 (19), 13-20.

Ballano, I. y Muñoz, I. (2010). Cómo elaboran sus trabajos escritos los universitarios. Claves para una realfabetización en la era digital. Universidad de Deusto. Recuperado de: http://reposital.cuaed.unam .mx:8080/jspui/handle/123456789/1520. Última fecha de consulta: 10/03/2015.

Burston, J. (2013). Mobile-Assisted Language Learning: a selected annotated bibliography of implementation studies (1994-2012), Language Learning \& Technology. Recuperado de: http://llt.msu.edu/issues/ october2013/burston.pdf. Última fecha de consulta: 4/03/2015.

Campos, J. F. (2013). Las TIC en la escritura académica (Trabajo de final de máster en Tecnología Educativa, e-learning y Gestión del Conocimiento). Universidad de Murcia, Ciudad Murcia.

Casanovas, M. (2014). La comunicación en la universidad: escritura académica y Tic. En J. F. Durán Medina (ed.), La era de las TIC en la nueva docencia. Londres: McGraw-Hill. 
Cassany, D. (2008). Leer_en_la_red. En E. Narváez y S. Cadena (Eds.). Los desafíos de la lectura y la escritura en la educación superior: Caminos posibles (pp. 195-219). Santiago de Cali: Universidad Autónoma de Occidente.

Cassany, D. (2011). En_línea. Leer y escribir en la red. Barcelona: Anagrama.

Castelló, M. (2009). Aprender a escribir textos académicos: ¿Copistas, escribas, compiladores o escritores? En J. I. Pozo y M. del Puy Pérez (Eds.), Psicología del aprendizaje universitario: La formación en competencias (pp.120-133). Madrid: Morata.

Coiro, J. (2003). Exploring literacy on the Internet. Reading comprehension on the Internet: Expanding our understanding of reading comprehension to encompass new literacies. The Reading Teacher, 56 (5), 458-464. Recuperado de: http://www.annenbergmedia.org/workshops/teachreading35/pdf/ read_comprehension_internet.pdf. Última fecha de consulta: 10/03/2015.

Dede, C. (2005). Planning for neomillenial learning styles: Implications for investments in technology and faculty. En D. Oblinger y J. Oblinger (Eds.). Educating the net generation. Boulder, CO: EDUCAUSE.

Emig, J. (1971). The composing processes of twelfth graders. Urbana, Illinois: National Council of Teachers of English.

Emig, J. (1983). The web of meaning: Essays on writing, teaching, learning, and thinking. Upper Montclair, NJ: Boynton/Cook.

Espuny, C., González, J., Lleixa, M., Gisbert, M. (2011). Actitudes y expectativas del uso educativo de las redes sociales en los alumnos universitarios. Revista de Universidad y Sociedad del Conocimiento, 8 (1), 171-185.

Fundación Telefónica (2014). Informe sobre el estado de las apps en España. Recuperado de: http://madrid. theappdate.com/vinformeestadoapps/. Última fecha de consulta: 4/03/2015.

Gómez, M., Roses, S., y Farias, P. (2011). El uso académico de las redes sociales en universitarios, Comunicar, 38 (19), 131-138.

Gutiérrez, A., Palacios, A., y Torrego, L. (2010). Tribus digitales en las aulas universitarias. Comunicar, 34, 173-181.

Iglesias, M. T., y Molina, S. (2013). Evolución de la competencia "gestión de la información" en el alumnado de Magisterio. Aula Abierta, 41 (3), 45-60.
Khan, S. A., Bhatti, R., y Khan, A. A. (2011). Use of ICT by students: A survey of faculty of education at IUB. Library Philosophy and Practice 2011. Recuperado de: http://unllib.unl.edu/LPP/khan-bhatti-khan.pdf. Última fecha de consulta: 4/03/2015.

Knobel, M., y Lankshear, C. (2014). Studying New Literacies. Journal of Adolescent \& Adult Literacy, 58(2), 97-101.

Leu, D. J., Kinzer, C. K., Coiro, J. L., y Cammack, D. W. (2004). Toward a theory of new literacies emerging from the internet and other information and communication technologies. En R. B. Ruddel y N. J. Unrau (Eds.), Theoretical models and process of reading (pp. 1570-1613). Newark, DE: International Reading Association.

Markauskaite, L. (2006). Towards an integrated analytical framework of information and communications technology literacy: From intended to implemented and achieved dimensions. Information Research, 11(3), 252-280.

Moje, E. B. (2000). Reframing adolescent literacy research for new times: Studying youth as a resource. Reading, Research and Instruction 41(3), 211-228.

Monereo, C., y Fuentes, M. (2005). Aprender a buscar y seleccionar en Internet. En C. Monero (Coord.), Internet y competencias básicas. Aprender a colaborar, a comunicarse, a participar, a aprender. Barcelona: Graó.

Monge, S., y Olabarri, M. E. (2011). Los alumnos de la UPV/-EHU frente a Tuenti y Facebook: usos y percepciones, Revista Latina de Comunicación Social, 66, 79-100. Recuperado de: http://www.revistalatinacs. org/11/art/925_UPV/04_Monge.html. Última fecha de consulta: 10/03/2015.

$\mathrm{Ng}, \mathrm{W}$. (2012). Can we teach digital natives digital literacy? Computers \& Education, 59(3), 1065-1078. Recuperado de: http://www.sciencedirect.com/ science/article/pii/S0360131512001005. Última fecha de consulta: 4/03/2015.

Nuzzaci, A. (Noviembre 14-15, 2013). The use of facebook in informal contexts: What Implication does it have for formal contexts? En SIREM 2013, ICT in Higher Education and Lifelong Learning. Università degli Studi di Bari, Bari.

Oliver, R., Towers, S., y Oliver, H. (2000). Information and communications technology literacy: Getting serious about IT. En J. Bourdeau y R. Heller (Eds.). Proceedings of World Conference on Educational 
Multimedia, Hypermedia and Telecommunications 2000 (pp. 862-867). Chesapeake, VA: Association for the Advancement of Computing in Education (AACE). Recuperado de: http://www.editlib. org/p/16174/. Última fecha de consulta: 20/03/2015.

Oliver, R., Towers, S., y Oliver, H. (2000). Information and communications technology literacy: Getting serious about IT. En J. Bourdeau y R. Heller (Eds.), Proceedings of World Conference on Educational Multimedia, Hypermedia and Telecommunications 2000 (pp. 862-867). Chesapeake, VA: Association for the Advancement of Computing in Education (AACE). Recuperado de: http://www.editlib. org/p/16174/ Última fecha de consulta: 10/03/2015.

Tapia A., Gómez B., Herranz de la Casa, J. M., y Matellanes, M. (2010). Los estudiantes universitarios ante las redes sociales: cuestiones de uso y agrupación en estructuras elitistas o pluralistas, Vivat Academia, 113. Recuperado de: http://www.ucm.es/info/-vivataca/numeros/n113/DATOSS.htm. Última fecha de consulta: 10/03/2015.

Tiana, A. (2011). Análisis de las competencias básicas como núcleo curricular en la educación obligatoria española. Bordón. Revista de Pedagogía, 63 (1), 63-75.
Togia, A. y Tsigilis, N. (2010). Awareness and use of electronic information resources by education graduate students: Preliminary results from the Aristotle University of Thessaloniki. En A. Katsirikou y C. $\mathrm{H}$. Skiadas, Qualitative and quantitative methods in libraries: proceedings of the International Conference on QQML2009 (pp. 464-472). Singapore: World Scientific. Recuperado de: http://www.worldscientific.com/doi/abs/10.1142/9789814299701_fmatter. Última fecha de consulta: 10/03/2015.

Tolar Burton, S., y Chadwick, S. A. (2000). Investigating the practices of student researchers: Patterns of use and criteria for use of internet and library sources. Computers and Composition, 17 (3), 309-328.

Ware, P. D., y Warschauer, M. (2005). Hybrid literacy texts and practices in technology-intensive environments. International Journal of Educational Research, 43(7), 432-445.

Wilber, D. J. (2009). College Students and New Literacy Practices. En J. Coiro, M. Knobel, C. Lankshear y D. Leu (Eds.), Handbook of Research on New Literacies (pp. 553-581). Nueva York: Routledge. 